PALEO

Revue d'archéologie préhistorique

$27 \mid 2016$

Varia

\title{
Nouvelle datation du « masque » de La Roche- Cotard (Langeais, Indre-et-Loire, France)
}

New dating of the " mask » of La Roche-Cotard (Langeais, Indre-et-Loire, France)

Jean-Claude Marquet, Michel Lorblanchet, Christine Oberlin, Edit ThamoBozso et Thierry Aubry

\section{(2) OpenEdition}

\section{Journals}

Édition électronique

URL : http://journals.openedition.org/paleo/3144

DOI : $10.4000 /$ paleo.3144

ISSN : 2101-0420

Éditeur

SAMRA

Édition imprimée

Date de publication : 30 décembre 2016

Pagination : 253-263

ISSN : 1145-3370

\section{Référence électronique}

Jean-Claude Marquet, Michel Lorblanchet, Christine Oberlin, Edit Thamo-Bozso et Thierry Aubry, «

Nouvelle datation du « masque » de La Roche-Cotard (Langeais, Indre-et-Loire, France) », PALEO [En ligne], 27 | 2016, mis en ligne le 01 juin 2018, consulté le 07 juillet 2020. URL : http://

journals.openedition.org/paleo/3144; DOI : https://doi.org/10.4000/paleo.3144

\section{(c) $(1) \&$}

PALEO est mis à disposition selon les termes de la licence Creative Commons Attribution - Pas d'Utilisation Commerciale - Pas de Modification 4.0 International. 


\title{
Nouvelle datation du « masque » de La Roche-Cotard (Langeais, Indre-et-Loire, France)
}

\author{
Jean-Claude MARQUET(a), Michel LORBLANCHET(b) ${ }^{(\mathrm{a}}$, Christine OBERLIN(c), \\ Edit THAMO-BOZSO(d) ${ }^{(\mathrm{d})}$ Thierry AUBRY(e)
}

\begin{abstract}
Résumé : Le « masque » de la Roche-Cotard a été découvert au sein d'une couche contenant une industrie lithique de technologie et typologie caractéristiques du Paléolithique moyen. Après une première datation de la couche, supérieure à $32 \mathrm{ka} \mathrm{BP}$ et deux autres datations par le ${ }^{14} \mathrm{C}$ attribuant à la couche un âge supérieur à $40 \mathrm{ka}$, une datation sur quartz par la méthode de la luminescence (OSL) donne un âge de 75,6 \pm 5,8 ka au dépôt alluvial qui contient le « masque ». Ce nouveau résultat confirme que les datations ${ }^{14} \mathrm{C}$ par Spectrométrie de Masse par Accélérateur (AMS) obtenues sur des fragments osseux de cette même couche sont au-delà de la limite de la validité de la méthode de datation par le radiocarbone. Nous exposons, en annexe, le principe de la méthode des datations OSL qui doivent être retenues pour dater l'occupation néandertalienne de La Roche Cotard II d'où provient le masque, et leurs implications.
\end{abstract}

Mots-clés : Paléolithique moyen, Neandertal, symbolique, datation luminescence.

Abstract: New dating of the "mask" of La Roche-Cotard (Langeais, Indre-et-Loire, France). The "mask" of La Roche-Cotard was discovered in a layer containing a characteristic Middle Paleolithic lithic industry. After a first ${ }^{14} \mathrm{C}$ result of $32 \mathrm{ka}$ BP, two further ${ }^{14} \mathrm{C}$ dates now assign the layer to more than $40 \mathrm{ka}$, but above all an OSL date obtained on quartz attributes to the layer containing the «mask» an age of $75.6 \pm 5.8 \mathrm{ka}$. This new result confirms that $14 \mathrm{C}$ dates with Accelerator Mass Spectrometry, obtained on bones of that layer are beyond the limit of validity of the radiocarbon dating method. We present the principle of the method of luminescence dating that should be retained for the Neanderthal occupation of the Roche Cotard II level 7 and its implications.

Key-words: Middle Palaeolithic, Neanderthal, Symbolic, Luminescence dating.

\section{Introduction}

Le site de La Roche-Cotard, découvert en 1912 grâce à l'extraction, en 1846, de matériaux pour les besoins de la mise en place de la voie ferrée Tours-Angers, a fait l'objet de fouilles au moment de sa découverte (d'Achon 1913), dans la seconde partie des années 1970 (Marquet 1979, Marquet et al. 1997; Lorblanchet 1999) et depuis 2008
(Marquet et Lorblanchet 2000, 2003). La fouille des années 1970 a permis la découverte, devant l'entrée de la grotte d'Achon, d'un épais dépôt sédimentaire mais de dimensions très limitées $(4 \mathrm{~m} \times 2 \mathrm{~m})$ comprenant notamment un lambeau de plage de Loire (fig. 1). Le sédiment de la couche 7c, la partie inférieure de la couche 7 , est un sable grossier comportant de gros fragments de chert et calcaire qui comble les irrégularités de la surface du

(a) Chercheur associé au Laboratoire Archéologie et Territoires Tours - jcmarquet@wanadoo.fr

(b) Directeur de recherches honoraire au CNRS

(c) Centre de Datation par le Radiocarbone UMR 5138 Archéométrie et Archéologie, 40 boulevard Niels Bohr, FR-69622 Villeurbanne cedex

(d) Geological and Geophysical Institute of Hungary, H-1143 Budapest, Stefánia 14.

(e) Fundação Côa Parque, P-Vila Nova de Foz Côa 


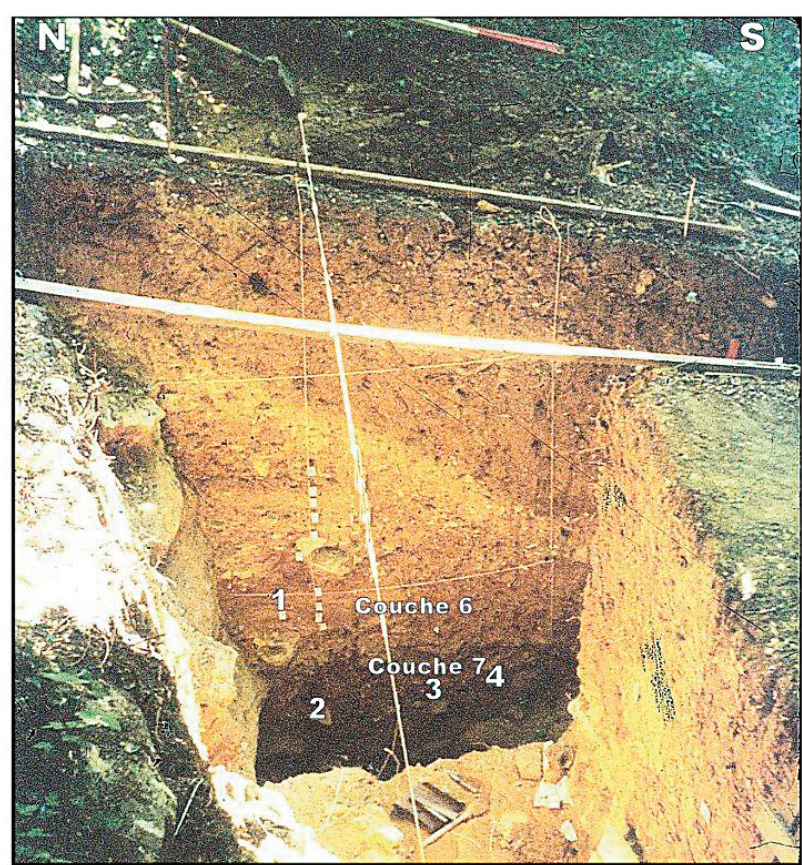

Figure 1 - Langeais La Roche-Cotard II. Stratigraphie et positionnement des quatre dernières datations réalisées. Les cordelettes de la coupe sagittale sont distantes de un mètre.

Figure 1 - Langeais. La Roche-Cotard II. Stratigraphy and position of the latest four dates obtained. The strings of the sagittal section are one meter apart.

substrat. Elle est surmontée par le même sable grossier (7b) mêlé de graviers disposés le plus souvent à plat et se termine par une couche très mince (7a), riche en grandes paillettes de mica. La couche 6 a une composition minéralogique un peu différente de la 7 mais il s'agit aussi d'un sable grossier surmonté, comme la couche 7 , par un sable fin homogène. Les couches 6 et 7 sont d'origine alluviale, mises en place lors de deux crues successives de la Loire (Macaire in Marquet et al. 1997 - p.76). C'est la couche 7 qui était le support d'un niveau archéologique contenant une industrie lithique caractéristique du Moustérien et de rares restes de faune, mais bien conservés. Les remontages entre vestiges lithiques de ce niveau indiquent qu'ils n'ont pas été affectés par des déplacements post-dépositionnels de grande ampleur (Marquet et al. 1997 - p. 87). Ce niveau comprenait également un objet composite longtemps appelé objet énigmatique (Marquet 1979) et dénommé «masque» de La Roche-Cotard par Michel Lorblanchet.

Le caractère intentionnel de la production de cet objet de $93 \mathrm{~mm}$ de hauteur, $105 \mathrm{~mm}$ de largeur, de $40 \mathrm{~mm}$ d'épaisseur et pesant $299 \mathrm{~g}$ (fig. 2) a été exposé précédemment (Marquet et Lorblanchet ibid). II repose sur quatre critères : même débordement de l'esquille osseuse de part et d'autre du pont rocheux du bloc support, blocage de l'esquille osseuse à l'intérieur du conduit naturel du bloc support à l'aide de petites plaquettes bien visibles sur l'objet et sur sa tomographie, enlèvement d'éclats sur chacune des

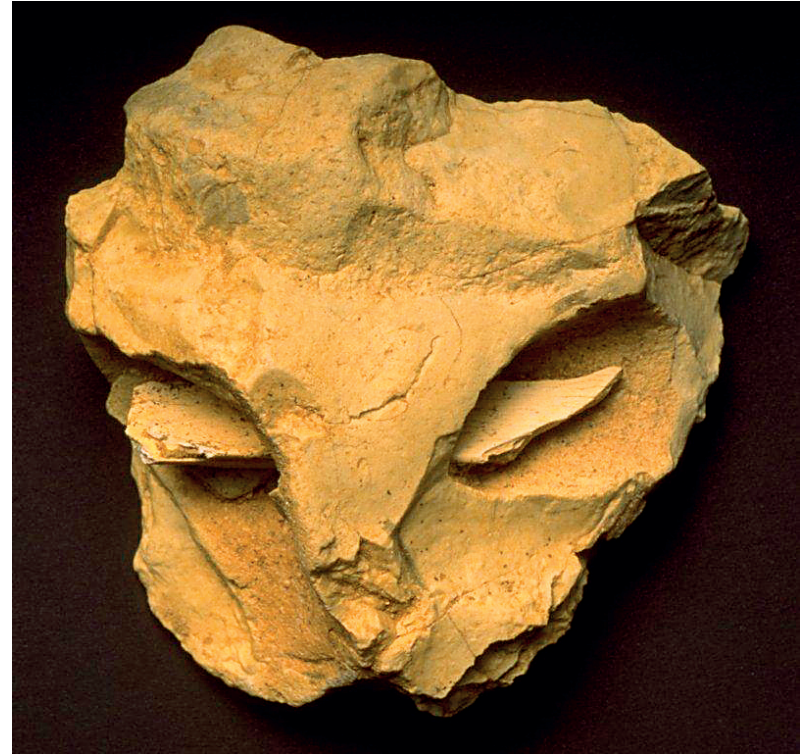

Figure 2 - Langeais. La Roche-Cotard II. Le « masque». Hauteur : $93 \mathrm{~mm}$, largeur : $105 \mathrm{~mm}$, épaisseur : $40 \mathrm{~mm}$, masse : $299 \mathrm{~g}$.

Figure 2 - Langeais. La Roche-Cotard II. The "mask". Height: $93 \mathrm{~mm}$, width: $105 \mathrm{~mm}$, thickness: $40 \mathrm{~mm}$, weight: $299 \mathrm{~g}$.

deux faces du bloc pour créer une meilleure symétrie du bloc, régularisation des deux arêtes dans la partie " inférieure » de l'objet par écrasement (le vocabulaire employé ici résulte du positionnement de l'objet choisi $a$ priori et tel que la face de la figure 2 serait la face antérieure).

II semble difficile de nier le caractère symbolique de cette production qui a été découverte au sein de la couche moustérienne. L'hypothèse de F. d'Errico (Nicoud 2004) selon laquelle l'objet aurait pu servir de poids pour tendre un vélum ne tient pas du fait de la très faible masse de l'objet (299 g) qui semble bien être aussi complet qu'au moment de son abandon dans la couche sableuse. L'hypothèse de P. Pettitt (2003) selon laquelle cet objet pourrait être le résultat d'un jeu d'enfant ne nous semble pas non plus convenir car si les deux premiers critères de caractérisation pourraient correspondre à cette possibilité (équilibre et calage), en revanche, il ne nous semble pas que les deux derniers puissent entrer dans ce cadre (recherche de symétrie et de régularité).

\section{1 - Datations de restes osseux par le radiocarbone}

Le 10 janvier 1980, G. Delibrias du Centre des faibles radioactivités de Gif-sur-Yvette (in litteris) indique un âge supérieur ou égal à 32,1 ka (GIF-4383 du 9-01-1980) pour une datation ${ }^{14} \mathrm{C}$ effectuée sur la grande côte d'herbivore trouvée à côté du foyer en cuvette de la couche 7 de La 
Roche-Cotard II, la couche qui a donné le «masque». Elle nous précise dans son courrier d'accompagnement que : "les datations très anciennes, sur la plupart des matériaux, sont peu fiables. Pour ces raisons, j'ai longtemps hésité à vous communiquer les résultats obtenus... Je pense qu'il ne faut considérer les âges mesurés que comme des âges apparents donnant une limite inférieure à l'âge réel'». C'est cette date qui a été donnée dans les publications qui ont précédé la reprise des fouilles en 2008.

À partir de 2009 , deux nouvelles datations ${ }^{14} \mathrm{C}$ ont été effectuées sur des os provenant toujours de la couche moustérienne ayant donné le «masque». Effectuées par le centre de datation par le radiocarbone de Villeurbanne, la première datation (Lyon-6 963/SacA 19 432) a indiqué un âge supérieur à $40 \mathrm{ka}$; la seconde datation (Lyon-9 086SacA 28 353) a donné également un âge supérieur à $40 \mathrm{ka}$. Ces deux datations ont été réalisées sur des os et dans les deux cas le collagène a été traité par ultrafiltration (Higham et al. 2011 ; Oberlin et al. 2015). Des âges plus précis que ceux indiqués ici ont été mesurés par le laboratoire mais étant donné que nous sommes en limite d'application de la méthode, le laboratoire n'a pas souhaité la publication de ces valeurs (tabl. 1) qui, bien que plus vieilles que l'âge obtenu en 1980, restent très en deçà des dates obtenues par la méthode OSL

\section{2 - Datation par Luminescence Stimulée Optiquement}

En 2014, nous avons procédé à la réouverture de La Roche-Cotard II, afin d'effectuer des prélèvements de sédiment dans les couches 7c (celle du «masque») et $6 f$ (couche alluviale située immédiatement au-dessus de la couche alluviale contenant le niveau moustérien (fig 3). Nous avons sollicité l'Institut géologique et géophysique de Hongrie (1145 Budapest Colombus u 17-23 1442 Budapest POBox 106) pour deux nouvelles datations OSL, d'autres échantillons ayant été, au préalable, mesurés pour les autres locus du site (tabl. 1).

La datation OSL est bien adaptée à la datation des sédiments puisqu'ils sont susceptibles d'avoir été blanchis lors de leur dépôt. La technique de l'aliquote unique (single aliquot) a été appliquée sur les échantillons de sédiments de La Roche Cotard. Elle permet de mesurer un grand nombre de fractions, supposées analogues, de l'échantillon à dater. La datation OSL des deux échantillons a été effectuée sur des grains de quartz de diamètre compris entre 0,10 et $0,16 \mathrm{~mm}$. Les protocoles de préparation et de mesures des échantillons ont été annexés à notre note.

\begin{tabular}{|c|c|c|c|c|c|c|}
\hline LRC I & & Méthode & Carré & Couche & Numérotation Labo & Date obtenue \\
\hline & Salle des lemmings & $14 \mathrm{C}$ & L 9 & c. supérieure & Lyon-6962 SacA 19431 & 39,5 à 35,4 ka calBC ou 41,4 à 37,3 cal $\mathrm{BP}$ \\
\hline & Salle des lemmings & $\mathrm{OSL}$ & L 9 & c. supérieure & MFGI/126.1 & $64,1 \pm 5,1 \mathrm{ka}$ \\
\hline & Extérieur cavité & OSL & $Y^{\prime} 08$ & c. moyenne & $\mathrm{MFGI}^{*} / 126.7$ & $72,6 \pm 6,0 \mathrm{ka}$ \\
\hline & Extérieur cavité & $14 \mathrm{C}$ & $Y^{\prime} 08$ & c. moyenne & Lyon 11276 (Sac A 37649) & $>40$ ka BP \\
\hline & Galerie moustérienne & $14 \mathrm{C}$ & D 14 & c. moyenne & Gif-4447 & $>38,4$ ka BP \\
\hline & Salle des lemmings & $14 \mathrm{C}$ & L 9 & c. moyenne & Lyon-6961 SacA 19430 & $>40 \mathrm{ka} \mathrm{BP}$ \\
\hline & Galerie moustérienne & $14 \mathrm{C}$ & 114 & c. moust. (c. 3) & Lyon-7864 SacA 23350 & $>40$ ka BP \\
\hline & Entrée & $14 \mathrm{C}$ & C 12 & c. inférieure & Lyon 11273 Sac A 37646 & $>40$ ka BP \\
\hline & Salle du pilier & $14 \mathrm{C}$ & Q 17 & c. 3 & Lyon-7865 SacA 23351 & $>45$ ka BP \\
\hline & Salle de la hyène & $14 \mathrm{C}$ & Y 21 & c. 1 & Lyon-10161 (SacA32828) & $>40$ ka BP \\
\hline \multirow{5}{*}{ LRC II } & Alluvions Loire & OSL & i 13 & $6 \mathrm{f}$ & MFGI/136.2 & $65,7 \pm 5,0 \mathrm{ka}$ \\
\hline & Couche moustérienne & OSL & i 13 & $7 \mathrm{c}$ & MFGI/136.1 & $75,6 \pm 5,8 \mathrm{ka}$ \\
\hline & Couche moustérienne & $14 \mathrm{C}$ & f. 12 & c. 7 & Gif-4383 & $>32,1 \mathrm{ka} \mathrm{BP}$ \\
\hline & Couche moustérienne & $14 \mathrm{C}$ & i 13 & c. 7 & Lyon-6963 SacA19432 & $>40$ ka BP \\
\hline & Couche moustérienne & $14 \mathrm{C}$ & h 12 & c. 7 & Lyon-9086 SacA 28353 & $>40$ ka BP \\
\hline \multirow[t]{4}{*}{ LRC III } & Remplissage abri & $14 \mathrm{C}$ & $?$ & c. XIII & Gif-4384 & $>45$ ka BP \\
\hline & Remplissage abri & $14 \mathrm{C}$ & 17 & c. XIII & Lyon-10162(SacA 32829) & $>45 \mathrm{ka} \mathrm{BP}$ \\
\hline & Remplissage abri & OSL & 17 & c. XIII & MFGI/126.3 & $56,1 \pm 4,5 \mathrm{ka}$ \\
\hline & Remplissage abri & $14 \mathrm{C}$ & $\mathrm{J} 6$ & c. $X$ & Lyon-10163(SacA 32830) & $>44$ ka BP \\
\hline \multirow[t]{9}{*}{ LRC IV } & Colluvion versant & $14 \mathrm{C}$ & 09 & c. $7 a$ & Lyon 9087 SacA 28354 & 31 à 29,5 ka calBC ou 32,6 à 31,1 calBP \\
\hline & Colluvion versant & OSL & Q 4/Q 5 & c. $7 \mathrm{c}$ & MFGI/126.4 & $48,1 \pm 3,8 \mathrm{ka}$ \\
\hline & Base colluvion versant & $14 \mathrm{C}$ & R 4 & c. 9 & Lyon-10160(SacA 32827) & $>44,6$ ka BP \\
\hline & Remplissage grotte-abri & OSL & R 3 & c. $12 \mathrm{~cd}$ & MFGI/126.6 & $86,2 \pm 6,5 \mathrm{ka}$ \\
\hline & Remplissage grotte-abri & $14 \mathrm{C}$ & Q 2 & c. $13 \mathrm{~b}$ & Lyon 7863 SacA 23349 & $>45 \mathrm{ka} \mathrm{BP}$ \\
\hline & Remplissage grotte-abri & $14 \mathrm{C}$ & Q 3 & c. $13 \mathrm{~d}$ & Lyon 7862 SacA 23348 & $>45$ ka BP \\
\hline & Remplissage grotte-abri & $14 \mathrm{C}$ & Q 3 & c. 15 & Lyon 9088 SacA42835 & $>40$ ka BP \\
\hline & Remplissage grotte-abri & $14 \mathrm{C}$ & Q 1 & c. $16 \mathrm{a}$ & Lyon 9089 (Sac A 28356) & $>40$ ka BP \\
\hline & Remplissage grotte-abri & OSL & Q 4 & c. 22 & MFGI/126.5 & $189,4 \pm 14,9 \mathrm{ka}$ \\
\hline
\end{tabular}

MFGI $^{*}=$ Magyar Földtani és Geofizika Intézet (Geological and Geophysical Instit of Hungary) H-1143 Budapest, Stefánia 14. Hungary.

Director : Dr. Tamas Francsik., Operator : Dr. Edit Thamo-Bozso.

Tableau 1 - Langeais. La Roche-Cotard. Datations par le radiocarbone et la méthode OSL.

Tableau 1- Langeais. La Roche-Cotard. Radiocarbon and OSL dates. 


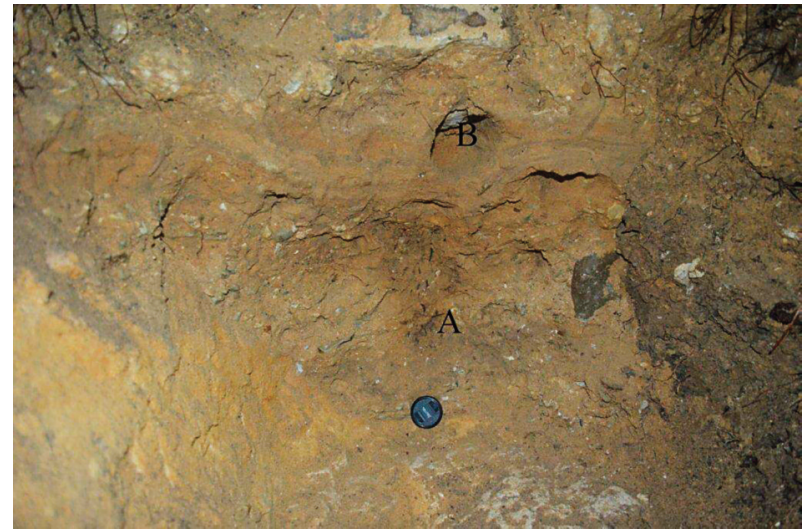

Figure 3 - Langeais. La Roche-Cotard.II. Situation des deux prélèvements pour datations OSL. En A, dans couche 7c; en B, dans couche $6 f$

Figure 3 - Langeais. La Roche-Cotard II. Positions of the two samples for OSL dating. A for level $7 c$ and B for level $6 f$.

L'âge retenu pour l'échantillon de la couche 6f (RSC / LSO / 9), identifié par le laboratoire sous le numéro MFGI / 136.2 , est de 65,7 $\pm 5,0 \mathrm{ka}$ (cf. annexe). Celui de la couche $7 \mathrm{c}$ contenant le niveau moustérien et le « masque » (RSC / LSO / 8), est de 75,6 \pm 5,8 ka. MFGI / 136.1.

\section{3 - Discussion}

Le site de La Roche-Cotard fait actuellement l'objet d'un projet collectif de recherches dont l'objectif principal est la détermination de sa chronologie, la compréhension de sa genèse géologique et de la nature des occupations anthropiques et animales de ses quatre locus. Des productions à caractère symbolique, probablement sans rapport entre elles, un objet dénommé " masque de La Roche-Cotard » trouvé au sein d'une couche moustérienne (LRC II), des tracés (digitaux et autres) ainsi que de très rares taches d'ocre rendent plus aiguë la nécessité de tenter d'atteindre l'objectif notamment chronologique. Le problème ne se trouve pas non plus simplifié du fait d'un certain isolement du site qui n'est entouré, pour ce qui est des gisements stratifiés contenant des industries du Paléolithique moyen, que par le site des Roches d'Abilly à $50 \mathrm{~km}$ au sud-est (Aubry et al. 2012, 2014a ; Aubry, Walter et Peyrouse 2014b), Roc-en-Pail à Chalonnes-sur-Loire à 60 km à l'ouest (Gruet 1984 ; Soriano 2012), le site d'Angé en Loir-et-Cher à 50 km à l'ouest (Locht et al. 2015). On ne citera que pour mémoire le gisement des Champs-Penais, encore sur la commune d'Abilly, dont la stratigraphie et le contenu archéologique sont mal connus (Cordier et Berthouin 1956).

En ce qui concerne La Roche Cotard, l'âge obtenu par OSL sur le sédiment de la base de la séquence de LRC IV $(189,4 \pm 14,9 \mathrm{ka})$ situe son dépôt contemporain de la fin du stade isotopique 7 ou bien du début du stade 6 (fig. 4), elle ancre solidement dans la durée la dynamique du remplissage. Bien que l'on entre alors dans un épisode glaciaire, il ne semble pas que nous ayons ici trace d'un colluvionnement, ce qui est tout à fait possible si le fleuve se trouve immédiatement en bas du versant, emportant les matériaux au fur et à mesure qu'ils s'accumulent. II est également possible qu'il y ait eu accumulation en l'absence du fleuve mais dégagement ensuite au retour de celui-ci.

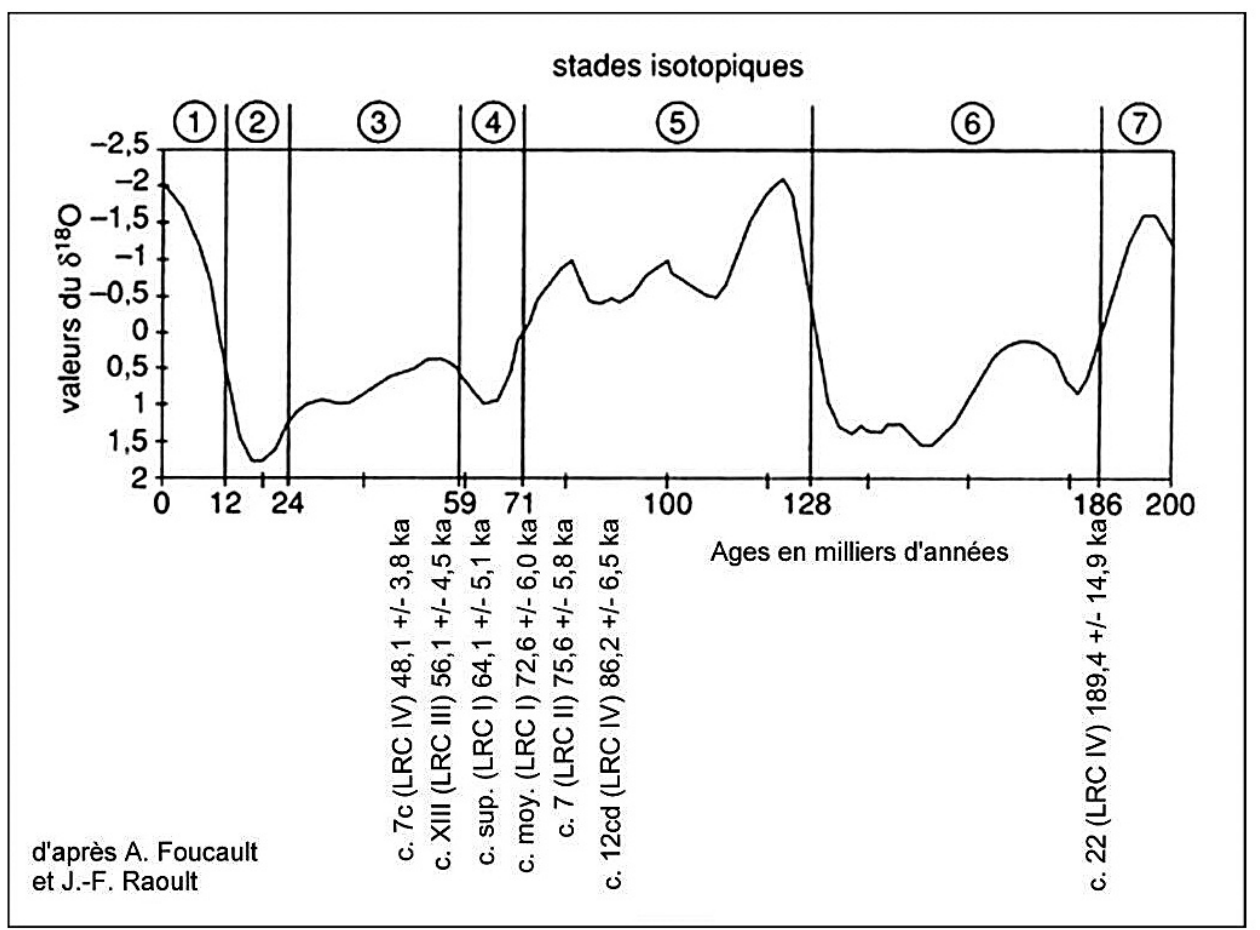

Figure 4 - Langeais. La RocheCotard. Positionnement de datations OSL sur la courbe de référence des variations du 0180 océanique pour les 200000 dernières années, corrigée d'après l'orbite terrestre (J. Imbrie et al. 1984).

Figure 4 - Langeais. La RocheCotard. Position of some OSL dates on the reference curve of oceanic 0180 variations for the last 200,000 years, corrected according to the earth's orbit (Imbrie et al. 1984). 
Au cours de l'Eemien, une importante phase d'altération augmente le volume des cavités donnant de volumineux blocs de craie tuffeau qui restent piégés, enrobés dans une matrice argileuse d'altération (LRC IV et salle la plus profonde de LRC I). Ensuite la cavité, désormais ouverte, est probablement alors fréquentée par les grands carnivores (ours des cavernes et grand lion des cavernes) dont on n'a retrouvé que de rares restes.

Les premières occupations humaines du site semblent alors celles de LRC IV (couche $12 \mathrm{c} / \mathrm{d}$ vers $85 \mathrm{ka}$ ) et à l'entrée de la grotte LRC I (lithique sous la couche d'inondation qui est datée de 72,6 $\pm 6,0 \mathrm{ka}$ ).

La couche 7 qui est le support du niveau moustérien de La Roche-Cotard II est, parmi les multiples strates qui appartiennent aux quatre locus du site, l'une de celles qui atteste, de la manière la plus caractéristique, un dépôt fluviatile de la Loire. D'autres dépôts issus du fleuve existent dans les autres locus mais on note ici la présence d'un lambeau d'une véritable plage sableuse de Loire qui se met en place alors que le versant a été déchargé de toutes ses colluvions par la sape du fleuve.

L'industrie lithique de la couche 7 a été produite presque exclusivement selon un principe de débitage Levallois à éclats récurrents centripètes (Boëda 1994), à partir de galets de silex du Turonien inférieur provenant de terrasses fluviales ou de silex crétacés ou cénozoïques, provenant de plusieurs sources régionales du bassin de la Loire et de ses affluents. Les éclats, à talon finement facetté et peu épais ont été obtenus sur des nucléus Levallois dont le volume débité possède une faible convexité. II est possible d'en reconstituer un grâce à un remontage entre deux éclats (fig. 5). Un seul nucléus correspondant, cassé accidentellement lors de sa mise en forme, a été découvert. L'outillage retouché est représenté par différents types de racloirs simples ou convergents, aménagés sur ce type de supports. D'après les matières premières employées, plusieurs semblent avoir été produits ailleurs que sur le secteur fouillé. En association avec ces outils retouchés, et à proximité immédiate du « masque ", a été découvert un racloir simple convexe, à double patine. À la différence du reste de l'industrie lithique, il a été retouché sur un éclat obtenu selon un schéma Levallois à éclat préférentiel (Boëda 1994 ; Delagnes, Jaubert, Meignen 2007) et en silex de provenance plus lointaine, du Turonien supérieur de la région du Grand-Pressigny (Indre-et-Loire)

Ce mode de débitage de grands éclats selon un schéma opératoire Levallois préférentiel qui abouti à des nucléus caractéristiques est connu pour de nombreuses séries de surface de Touraine, du Poitou et de la Brenne, parfois associées à quelques bifaces (Primault 2003 ; Aubry et Rigaud 2004 ; Aubry, Walter, Pérouse 2014b). La chronologie de ces industries ne peut s'appuyer que sur les grands éclats Levallois produits selon ce principe de débitage qui ont été découverts en place dans la couche 5 du secteur LRA Virage 31/35 du site des Roches d'Abilly. Cette couche a fait l'objet de mesures par plusieurs méthodes de la luminescence sur des grains de quartz et

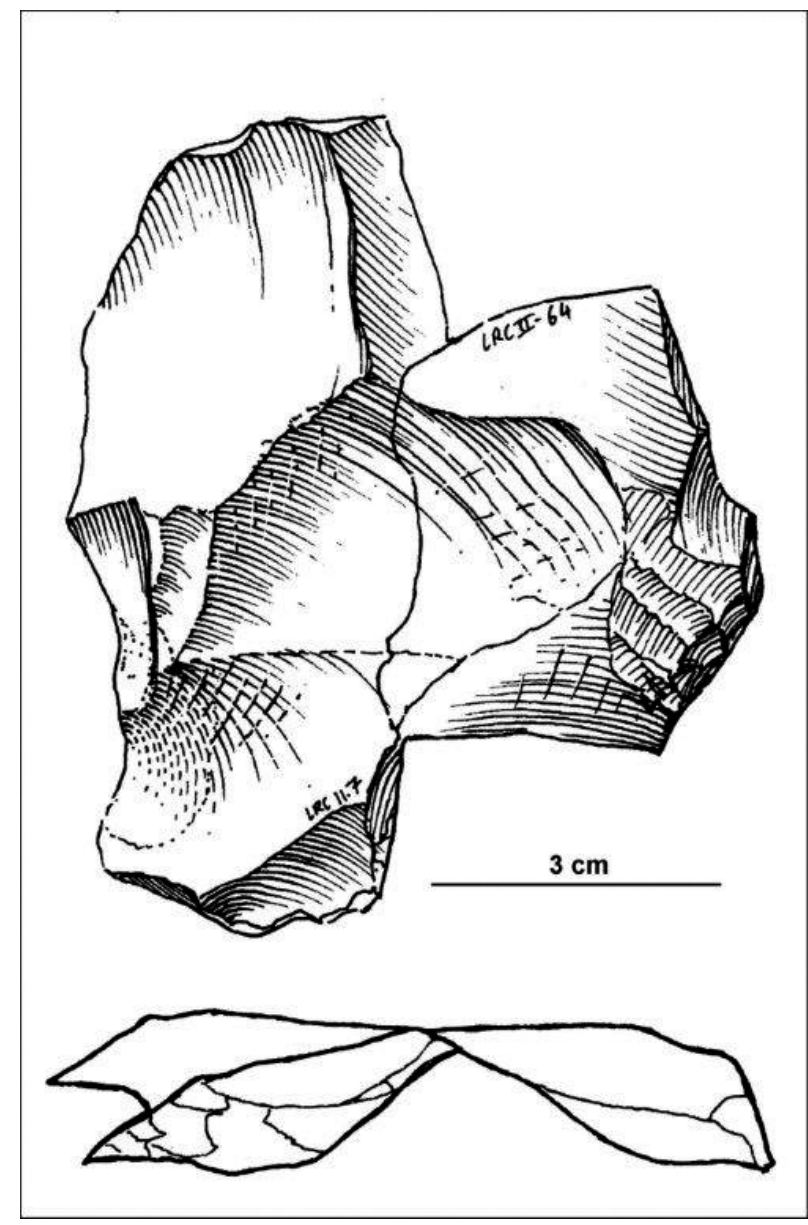

Figure 5 - Langeais. La Roche-Cotard II. Remontage de deux éclats obtenus selon un schéma Levallois récurrent centripète de la couche 7 .

Figure 5 - Langeais. La Roche-Cotard II. Refitting of two Levallois flakes from the level 7.

de feldspath (OSL). Deux des méthodes fournissent des résultats préliminaires qui sont cohérents et placeraient cette occupation entre 58 et 76 ka (Aubry, Walter, Peyrouse 2014b), en accord avec l'écart statistique de l'âge retenu pour la couche 7 de La Roche Cotard II et celui de la couche 6 qui est directement subjacente.

La méthode Levallois à éclat préférentiel est attestée dans le nord de la France « dans des assemblages moustériens plutôt anciens, antérieurs à la dernière période glaciaire» (Delagnes, Jaubert, Meignen 2007 - p. 216). Les nouvelles données obtenues à La Roche Cotard et aux Roches d'Abilly confirment cette hypothèse et une attribution à la fin du stade isotopique 5. Néanmoins, la chronologie et la contemporanéité des nombreuses séries utilisant un débitage Levallois à grands éclats préférentiels du nord et du centre-ouest de la France restent des hypothèses.

Le lithique, découvert par d'Achon en 1912 dans la première partie de la grotte (LRC I) est malheureusement 
introuvable aujourd'hui. D'après ce que l'on peut observer des bifaces des clichés dont on dispose de cette industrie est selon toute vraisemblance à rattacher à un Moustérien de tradition acheuléenne (MTA) et au stade isotopique 3. En effet, ces derniers se distinguent technologiquement et typologiquement des pièces bifaciales du site d'Angé (Loiret-Cher) qui a été attribué au stade $5 a$, trouvées dans un contexte pédo-sédimentaire de sol gris forestier (Locht et al. 2015). L'attribution à un faciès récent du Paléolithique moyen de cette occupation de La Roche Cotard I a trouvé une confirmation supplémentaire par la découverte d'un fragment d'un biface triangulaire dans une salle plus éloignée de l'entrée, lors des fouilles postérieures à 2008 (fig. 6).

D'un point de vue technologique, quelques éclats provenant de la couche $12 \mathrm{c} / \mathrm{d}$ de La Roche-Cotard IV indiquent une production selon le même schéma opératoire Levallois de type récurrent centripète que celui qui est majoritaire dans la couche 7 de La Roche-Cotard II.

Par contre, le petit ensemble de la base du remplissage de La Roche-Cotard III qui a permis un taux important de remontage, révèle une production d'éclats selon un schéma de débitage Discoïde à partir de galets de silex. Ce mode de production bien qu'ubiquiste au long du Paléolithique moyen (Thiébaut 2007 ; Jaubert 2010, Jaubert et al. 2011 ; Mourre 2003) est attesté dans la région, autour de $45 \mathrm{ka}$, dans la couche D2 de l'abri Bordes-Fitte (Aubry et al. 2014a). Les dates obtenues par OSL indiquent que cette occupation de La Roche Cotard III doit correspondre à la phase d'occupation la plus récente du site pendant le Paléolithique moyen.

\section{Conclusions}

Les travaux sur le site de La Roche Cotard s'inscrivent dans une tendance actuelle de la recherche qui accumule les indices d'activités symboliques très anciennes, largement antérieures à l'arrivée de l'Homme moderne, notamment chez les Néandertaliens.

Au début des années 1990, des spéléologues avaient découvert dans la vaste caverne de Bruniquel (Tarn et Garonne) à $300 \mathrm{~m}$ de l'entrée, c'est-à-dire dans la zone obscure profonde, une étrange structure ovalaire fermée de $7 \mathrm{~m} \times 4 \mathrm{~m}$ constituée d'un amas de stalagmites et concrétions diverses. À l'extrémité sud se distingue également un autre anneau de concrétions cassées, de forme ronde, d'environ $2 \mathrm{~m}$ de diamètre. À proximité, les spéléologues avaient remarqué la présence de boulettes d'argiles projetées au plafond de la galerie (Rouzaud, Soulier, Lignereux 1996). Ces vestiges ne sont sans doute pas ceux d'un habitat traditionnel bien que la structure puisse évoquer le fond de cabane moustérien de Molodova (Ukraine) dont la toiture en peaux était maintenue à la base par un grand anneau formé d'ossements de mammouths. Aucun outil, ni véritables traces d'occupation n'ont été pour l'instant identifiés dans cette structure. Cependant, un os d'ours des cavernes brûlé (trace probable d'un point d'« éclairage ») avait été daté au radiocarbone de 47,6 ka BP ce qui correspondait aux limites ultimes de la méthode de datation au carbone 14. De nouvelles datations à I'Uranium-Thorium viennent d'être réalisées sur des stalagmites scellenat ou constituant la grande structure. Le résultat obtenu de 176,5 ka situe l'édification de la structure dans une période correspondant à une phase ancienne du Paléolithique moyen (Jaubert et al. 2016).

Des recherches nouvelles, et peut-être des fouilles, préciseront sans doute ces premières données, mais d'ores et déjà la découverte de Bruniquel montre la pénétration des profondeurs obscures des grottes par les Moustériens maîtrisant l'éclairage et l'utilisation des cavités pour des activités très probablement rituelles, c'est-à-dire non directement liées à des impératifs immédiats de la vie quotidienne.

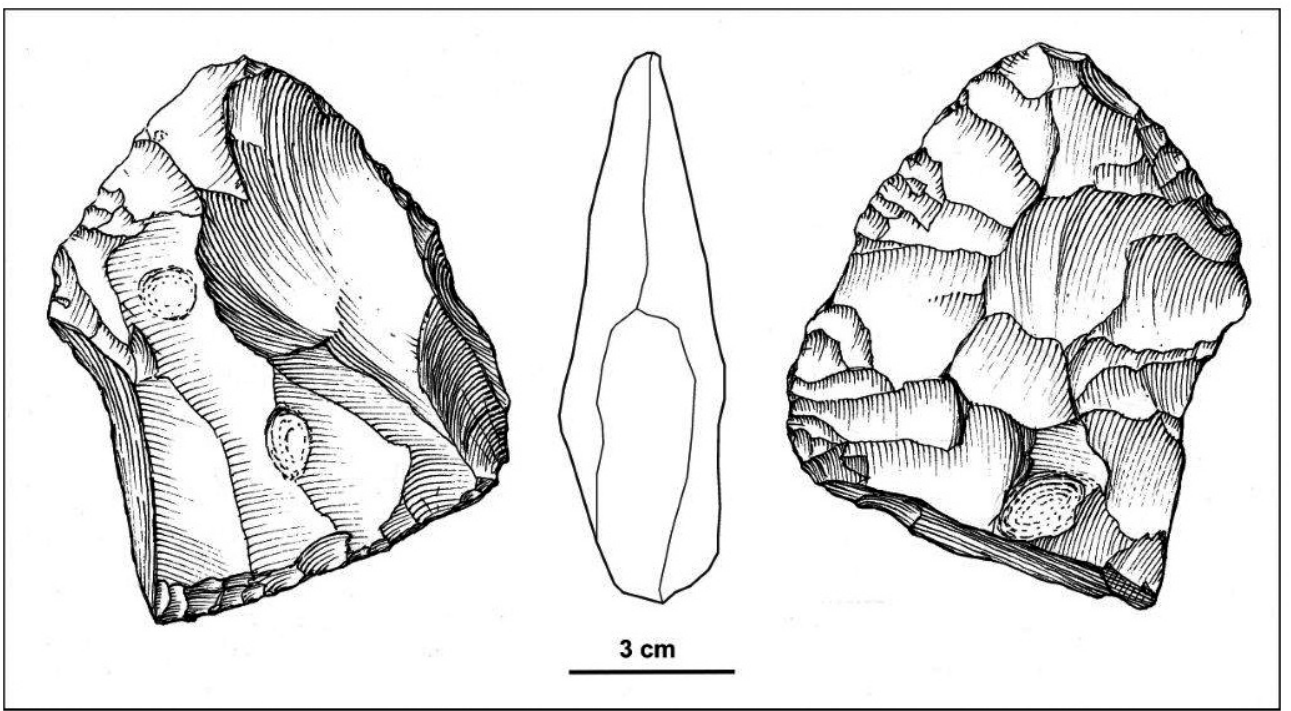

Figure 6 - Langeais. La Roche-Cotard I. Salle du pilier. Biface triangulaire.

Figure 6 - Langeais. La Roche-Cotard I. Pillar room. Triangular biface. 
En 2011, une main négative rouge de la grotte du Castillo (Cantabrie, Espagne) a été datée d'un minimum de 37630 $\pm 340 \mathrm{BP}$, par la méthode de datation U-Th du film de calcite recouvrant les peintures. Cette méthode a été mise au point par une équipe européenne de chercheurs comprenant des physiciens, des géologues et des préhistoriens (laboratoire CENIEH de Burgos). Une grosse ponctuation rouge de la même cavité, a été datée par la même méthode entre $34250 \pm 170$ et $37720 \pm 260$ Certaines dates associées à des motifs rouges de la grotte d'Altamira atteindraient même 41000 ans. Ces dates et cette méthode posent l'hypothèse des premières interventions pariétales par les hommes de Néandertal (Pike et al. 2012 ; Pike and Pettitt 2012).

En 2016, une nouvelle étude du bloc à cupules de la sépulture moustérienne $\mathrm{n}^{\circ} 6$ de La Ferrassie (Dordogne) fouillée par L. Capitan et D. Peyrony (Peyrony 1934) a confirmé et développé les observations des premiers fouilleurs. En utilisant des relevés photogrammétriques (avec des courbes de niveau équidistantes de $1 \mathrm{~mm}$ ) et des expérimentations, nous avons montré que ce bloc de calcaire coniacien recouvrant la sépulture d'un enfant néandertalien comportait en réalité 30 cupules sur sa face inférieure tournée vers le squelette. Ces cupules artificielles ont probablement été obtenues par des outils différents (galets et pointes) à des moments peut-être différents, ce qui étaye l'hypothèse d'opérations rituelles collectives en relation avec une sépulture (Lorblanchet et Bahn 2017). Ajoutons enfin, le motif abstrait gravé sur le bedrock de la grotte de Gorham's Cave à Gibraltar dont les traces profondes sont restées couvertes par un niveau archéologique moustérien qui confère ainsi à ces traces un âge supérieur à 39 ka. (Rodriguez-Vidal et al. 2014).

Ainsi l'existence d'une expression symbolique il y a 50000 ans, voire au-delà, précédant largement le Paléolithique supérieur et une origine probable de l'art rupestre européen au Paléolithique moyen ne cessent de se préciser. II apparait même de plus en plus, que dans d'autres régions du monde, les activités symboliques sont antérieures au Paléolithique moyen (Lorblanchet et Bahn ibid).

Dans ce sens, les nouvelles datations à $75 \mathrm{ka}$ (couche $7 \mathrm{c} / \mathrm{A}$ de la fig. 3) et $65 \mathrm{ka}$ (couche 6f/B de la même figure) complètent la série existante (tabl. 1) et confirment, en la précisant considérablement, l'ancienne datation obtenue sur os, par le radiocarbone, en 1980 qui indiquait un âge supérieur à 32,1 ka pour la couche 7 .

Avec l'obtention d'un faisceau de dates OSL, la compréhension de la nature et des rythmes de l'occupation de La Roche-Cotard a été enrichie chronologiquement. De nouvelles datations sont en cours qui vont probablement contribuer à éclairer encore un peu plus la chronologie de ce site complexe mais, quoi qu'il en soit, il convient maintenant de considérer que le «masque» de La RocheCotard provient d'une couche moustérienne qui a été datée par la méthode OSL d'un âge de l'ordre de $75 \mathrm{ka}$.

\section{Remerciements}

Nous remercions Francine Fellrath-Bacart pour la traduction en français de la contribution d'Edit ThamoBozso ainsi que les rapporteurs anonymes de la revue qui ont permis d'apporter des précisions au texte.

\section{Références bibliographiques}

ACHON (d') F. 1913 - Au pays des châteaux. La RocheCotard. Sa grotte préhistorique. La Touraine $1^{\text {ère }}$ année, $\mathrm{n}^{\circ} 4$.

AUBRY T., RIGAUD A. 2004 - Les industries Levallois sur éclats en Brenne. In : P. Paillet (dir.) Catalogue de l'exposition : "Préhistoire du Val de Creuse en Berry", Argenton, p. 35-36.

AUBRY T., DIMUCCIO L.-A., ALMEIDA M., BUYLAERT J.-P., FONTANA L., HIGHAM T., LIARD M., MURRAY A., NEVES M.-J., PEYROUSE J.-B., WALTER B. 2012 Stratigraphic and technological evidences from the Middle Palaeolithic-Châtelperronian-Aurignacian record at the Bordes-Fitte Rockshelter (Les Roches d'Abilly site, Central France) Journal of Human Evolution, vol. 62, p. 116-137.

AUBRY T., DIMUCCIO L.A., BUYLAERT J.P., LIARD M., MURRAY A.S., JØRKOV THOMSEN K., WALTER B. 2014a - Middle-to-Upper Palaeolithic site formation processes at the Bordes-Fitte rockshelter (Central France). Journal of Archaeological Science, 52, p. 436-457.

AUBRY T., WALTER B., PEYROUSE J.-B. 2014b Paléolithique moyen et supérieur de la vallée de la Claise : bilan de vingt ans d'études et nouvelles perspectives. Bulletin des Amis du Musée de Préhistoire du GrandPressigny, nº 65, p. 9-29.

BOËDA E. 1994 - Le concept Levallois : variabilité des méthodes. Monographie du CRA $n^{\circ}$ 9, Paris, CNRS Editions, $280 \mathrm{p}$.

CORDIER G., BERTHOUIN F. 1956 - L'abri Reignoux. Abilly (Indre-et-Loire), In: Congrès préhistorique de France, Compterendu de la $\mathrm{XV}^{\mathrm{e}}$ session, Poitiers-Angoulême : p. 97-100.

DELAGNES A., JAUBERT J., MEIGNEN L. 2007 - Les techno-complexes du Paléolithique moyen en Europe occidentale dans leur cadre diachronique et géographique. In : Les Néandertaliens : Biologie et culture. Paris, Éditions du CTHS (Documents préhistoriques, 23), p. 213-229.

GRUET M. 1984 - L'apport de deux sites angevins à la chronologie des terrasses fluviatiles : Roc-en-pail en Chalonnes-sur-Loire et Port-Launay sur la Sarthe. Bulletin de l'association française pour l'étude du Quaternaire, 1984 $(1,2,3)$, p. 13-18.

HIGHAM T. 2011 - European Middle and Upper Palaeolithic radiocarbon dates are often older than they look: problems with previous dates and some remedies. Antiquity 85 (327), p. 235-249. 
IMBRIE J., HAYS J.D., MARTINSON D.G., McINTYRE A., MIX A.C., MORLEY J.J, PISIAS N.G., PRELL W.L., \& SHACKLETON N.J., 1984 - The orbital theory of Pleistocene climate: support from a revised chronology of the marine d180 record. In: A.L. Berger, J. Imbrie, J. Hays, G. Kukla \& B. Saltzman (eds.), Milankovitch and Climate. Part I, Reidel Publ. Comp., Dordrecht, p. 269-305.

JAUBERT J. 2010 - Le Moustérien ou les sociétés néandertaliennes du Paléolithique moyen en France. In : Clottes, J. (éd.), La France préhistorique, un essai d'histoire. Paris, Gallimard, p. 64-92.

JAUBERT J., BORDES J.G., DISCAMPS E., GRAVINA B. 2011 - A New Look at the end of the Middle Palaeolithic Sequence in Southwestern France. In: Derevianko, Shunkov (ed.), Characteristic Features of the Middle to Upper Paleolithic Transition in Eurasia. Novosibirsk, Asian Palaeolithic Association, p. 102-115.

JAUBERT J., VERHEYDEN S., GENTY D., SOULIER M.,HAI CHENG, BLAMART D., BURLET C., CAMUS H., DELABY S., DELDICQUE D.,EDWARDS L. R., FERRIER C. LACRAMPE-CUYAUBERE F., LEVEQUE F., MAKSUD F., MORA P., MUTH X., REGNIER E., ROUZAUD J.-N., SANTOS F. 2016 - Early Neandertal constructions deep in Bruniquel Cave in south-western France. Nature, le 25 mai 2016. DOI:10.1038/nature18291.

LOCHT J.-L., COUTARD S., SORESSI M., KIEFER D., KOEHLER H., DEBENHAM N. 2015 - Angé (Loir-et-Cher) : un site moustérien à influences multiples. Mémoire 59 de la Société Préhistorique française. "Les plaines du NordOuest : carrefour de l'Europe au Paléolithique moyen ? » ch. 6, p. 101-125.

LORBLANCHET M. 1999 - La Naissance de l'art. Genèse de l'art préhistorique. Errance.

LORBLANCHET M. et BAHN P. 2017- " The First Artists; in search of the world's oldest art », 260 p. Thames and Hudson, à paraître en 2017.

MARQUET J.-C. 1979 - Le site paléolithique moyen de La Roche-Cotard, commune de Langeais (Indre-et-Loire) Congrès Préhistorique de France $21^{\mathrm{e}}$ Session MontaubanCahors, 1979, Vol. 2, p. 189-206.

MARQUET J.-C., AUBRY T., BYRNE N., DELPECH F., DESSE G., MACAIRE J.-J., PRAT F., RAGE J.-C., URBAN B., VISSET L. 1997 - Le site préhistorique de La Roche-Cotard à Langeais (Indre-et-Loire). Ed. CLD Chambray-les-Tours, 127 p., 82 fig, 24 tabl., 6 pl. hors texte.

MARQUET J.-C., LORBLANCHET M. 2000 - Le masque moustérien de La Roche-Cotard, Langeais (Indre-et-Loire), Paleo, n० 12, p. 325-338.

MARQUET J.-C., LORBLANCHET M. 2003 - A Neandertal face ? The protofigurine from La Roche-Cotard, Langeais (Indre-et-Loire, France) 》, Antiquity volume 77, $\mathrm{n}^{\circ}$ 298, p. 661-670.
MOURRE V. 2003 - Discoïde ou pas discoïde ? Réflexions sur la pertinence des critères techniques définissant le débitage discoïde. In : Discoid lithic technology: Advances and implications, ed. M. Peresani, British Archaeological Reports International Series 1120, p. 1-18.

NICOUD L. 2004 - « L'art néandertalien : réalité et énigme ». Archéologia, 407, Janvier 2004.

OBERLIN C., VALLADAS H. 2015 - Datation par le Radiocarbone de la fraction organique de l'os, In : Balasse M., Brugal J.-P., Dauphin Y., Geigl E.-M., Oberlin C. et Reiche I, (Eds.), Message d'os. Archéométrie du squelette animal et humain. Editions des archives contemporaines, Collection Sciences Archéologiques, Paris, p. 287-306.

PETTITT P. 2003 - Is this the infancy of art? Or the art of an infant? A possible Neanderthal face from La Roche-Cotard, France. Before Farming (Western Academic \& Specialist Press) 11 (3).

PEYRONY D. 1934 - « La Ferrassie », Préhistoiret. 3, p.1-92.

PIKE A.W.G., HOFFMANN D.L., GARCIA-DIEZ M., PETTITT P., ALCOLEA J., BALBIN R. de, GONZALESSAINZ G., LAS HERAS C., LAS HERAS J.A., MOTES R. et ZILHAO J. 2012 - U-Series Dating of Palaeolithic Art in 11 caves in Spain, SCIENCE, vol. 336, 15, june 2012, p. $1409-1413$.

PIKE A.W.G. and PETTITT P. 2012 - Redating Ice Age Art; were Neandertals the first artists in Europe. Current World Archaeology, Issue 55, p. 22-27.

PRIMAULT J. 2003 - Exploitation et diffusion des silex de la région du Grand-Pressigny au Paléolithique, thèse de l'université de Paris 10-Nanterre, 358 p.

RODRIGUEZ-VIDAL J., d'ERRICO F., ,GILES PACHECO F., BLASCO R., ROSELL J., JENNINGS R. P., QUEFFELEC A., FINLAYSON G., DARREN A.,GUTIERREZ LOPEZ J. M., CARRION J. S., NEGRO J. J., FINLAYSON S., CACERES L. M., BERNAL M. A., FERNANDEZ JIMENEZ S., FINLAYSON C. 2014 - A rock engraving made by Neandertals in Gibraltar. Proceedongs of the National Academy os Science of the United States of America (PNAS) vol. $111 \mathrm{n}^{\circ} 37$, p. $13301-13306$.

ROUZAUD F., SOULIER M., LIGNEREUX Y. 1996 - La grotte de Bruniquel (Tarn et Garonne), Spelunca $n^{\circ} 60$, p. 27-34.

SORIANO S. 2012 - Roc-en-Pail (Chalonnes-sur-Loire, 49). Une séquence de référence pour le Paléolithique moyen du Centre-Ouest. Demande d'opération archéologique. Dossier scientifique. Ministère de la Culture/DRAC Pays-de-la-Loire/Service régional de l'archéologie. 49 pages.

THIÉBAUT C. 2007 - Le Moustérien à denticulés des années 1950 à nos jours : définitions et caractérisation. Bull. Soc. Préhist. Fr. 104 (3), p. 461-481. 


\section{Annexe}

\section{Sample preparation}

OSL dating of two samples was carried out on quartz grains between 0.10 and $0.16 \mathrm{~mm}$ in diameter. Sample preparation was done in dark room under subdued red light conditions. Organic material was removed by $20 \% \mathrm{H}_{2} \mathrm{O}_{2}$, carbonates were dissolved by $10 \% \mathrm{HCl}$. Feldspars and the outer $10 \mu \mathrm{m}$ layer from the quartz grains (which absorbed a dose from alpha radiation) were removed by $40 \% \mathrm{HF}$ for 60 minutes (Aitken 1998). Then the quartz fractions were cleaned by $10 \% \mathrm{HCl}$, and the grains were mounted on stainless-steel discs in a $5 \mathrm{~mm}$ diameter (medium aliqouts) monolayer using silicone spray.

\section{OSL measurements and results}

OSL measurements were made using a Risø TL/OSL DA-15C/D automatic reader with a calibrated $90 \mathrm{Sr} / 90 \mathrm{Y}$ beta source. Blue light emitting diodes (LEDs, = $470 \pm 20 \mathrm{~nm}$ ) were used for the optical stimulation of quartz for $40 \mathrm{~s}$ at $125^{\circ} \mathrm{C}$. The resulted OSL signals were collected through an UV filter (Hoya U-340). Single-Aliquot Regenerative-dose (SAR) protocol was used to estimate equivalent doses with illumination for $40 \mathrm{~s}$ at $280^{\circ} \mathrm{C}$ in the last step of each cycle (Wintle and Murray 2006).

The quartz separates gave bright luminescence and their signals decreased rapidly (fig. 7). Infrared stimulation test indicated that the measured mineral separates were pure quartz. According to the preheat plateau test and thermal transfer test $260^{\circ} \mathrm{C}$ preheat temperature and $200^{\circ} \mathrm{C}$ cutheat were applied, when the thermal transfer is negligible, maximum $0.085 \%$ of the natural dose. Dose recovery ratio of the samples is $0.98 \pm 0.01$ in average, indicating that the SAR protocol correctly measures the given dose. Figure 8 shows the dose response growth curves of the samples. Only a few measured subsamples (aliquots) were saturated (3 for sample 161.1 and only one for 161.2) and not used for dating.

Equivalent doses $\left(D_{e}\right)$ were measured on 33 subsamples (aliquots) of sample 136.1. and 33 subsamples of sample 136.2. and showed more or less symmetric distribution (fig. 9), therefore the luminescence ages were calculated by the average $D_{e}$ value of each sample $(156.15 \pm 2.89$ Gy for sample 136.1. in 7c layer and $138.18 \pm 2.50$ Gy for sample 136.2. in $6 f$ layer, Tabl. 2).

Current and saturated water content of the samples was measured; for sample age dating we used the average value (10 and $13 \%$ water content). Dose rates of the sediments were calculated based on laboratory highresolution gamma spectrometry measurement (Canberra GC3020) of bulk samples using the conversion factors of Adamiec and Aitken (1998). In the case of medium water content the dose rates are $2.06 \pm 0.15 \mathrm{~Gy} / \mathrm{ka}$ for sample 136.1. and 2,10 $\pm 0.15 \mathrm{~Gy} / \mathrm{ka}$ for sample 136.2 (tab., Thamó-Bozsó et al. 2015).

The age of the sample from layer 6f (RSC / LSO / 9), identified by the laboratory under the number MFGI / 136.2, was $65.7 \pm 5.0 \mathrm{ka}$ (Tabl. 2). The age of the sample from layer 7c , the one containing the Mousterian level and the « mask » (RSC / LSO / 8), identified by the laboratory as MFGI / 136.1, was $75.6 \pm 5.8 \mathrm{ka}$ (tabl. 2).
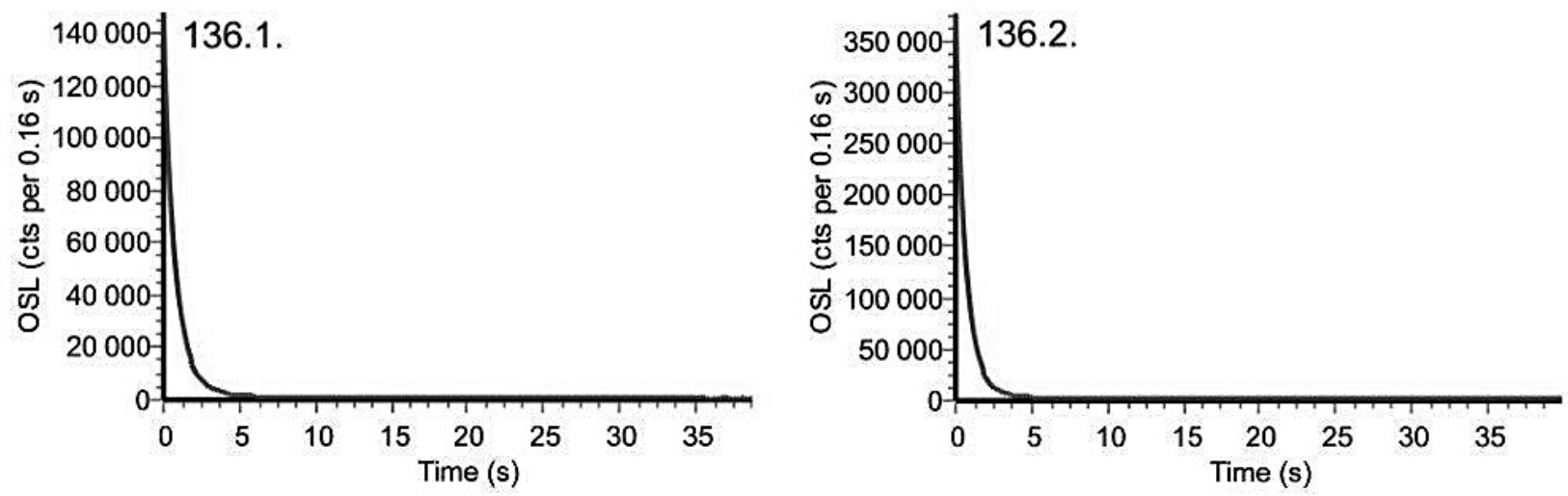

Figure 7 - Langeais. La Roche-Cotard II. Signaux OSL pour les échantillons 136.1 et 136.2.

Figure 7 - Langeais. La Roche-Cotard II. OSL Signals 136.1 and 136.2. 


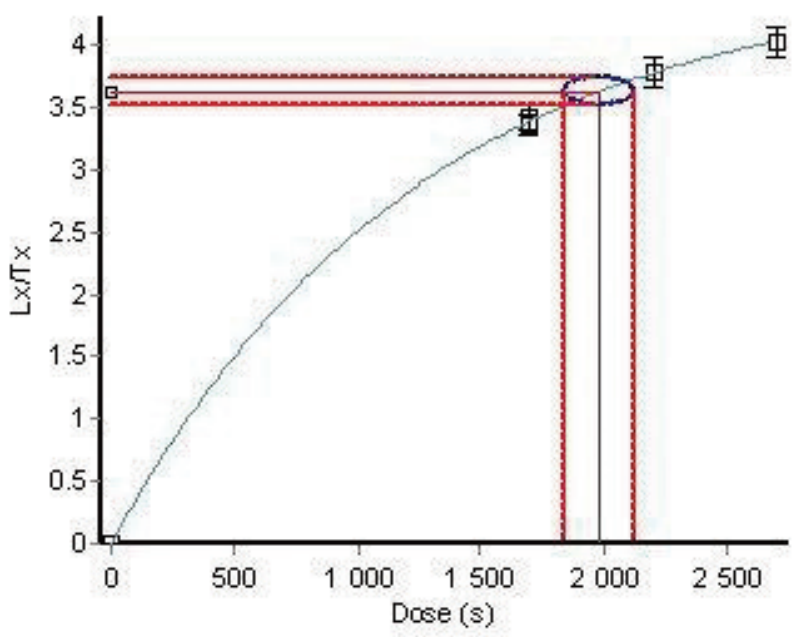

136.1 .

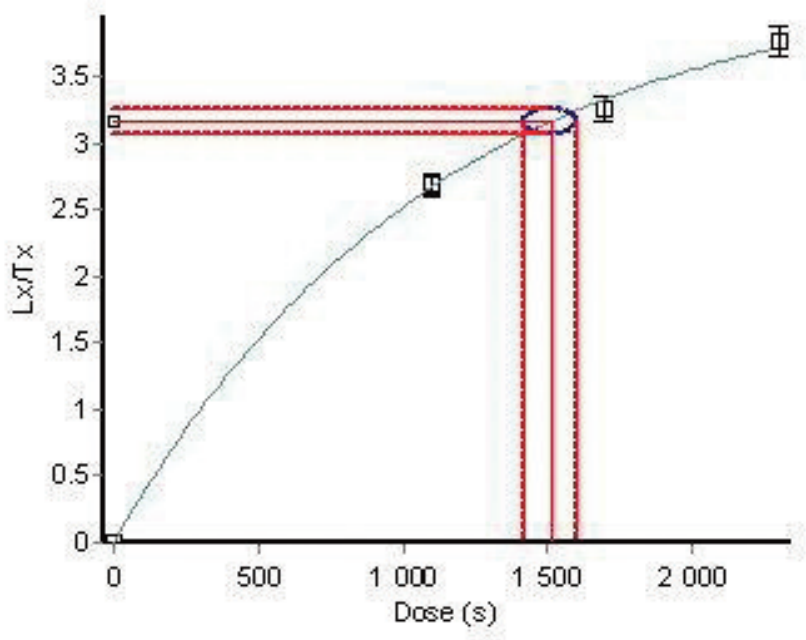

136.2 .

Figure 8 - Langeais. La Roche-Cotard II. Courbes de croissance OSL pour les échantillons 136.1 (calculée sur 36 aliquots) et 136.2 (calculée sur 34 aliquots).

Figure 8 - Langeais. La Roche-Cotard II. OSL growth curves 136.1 (calculated on 36 aliquots) and 136.2.(calculated on 34 aliquots).
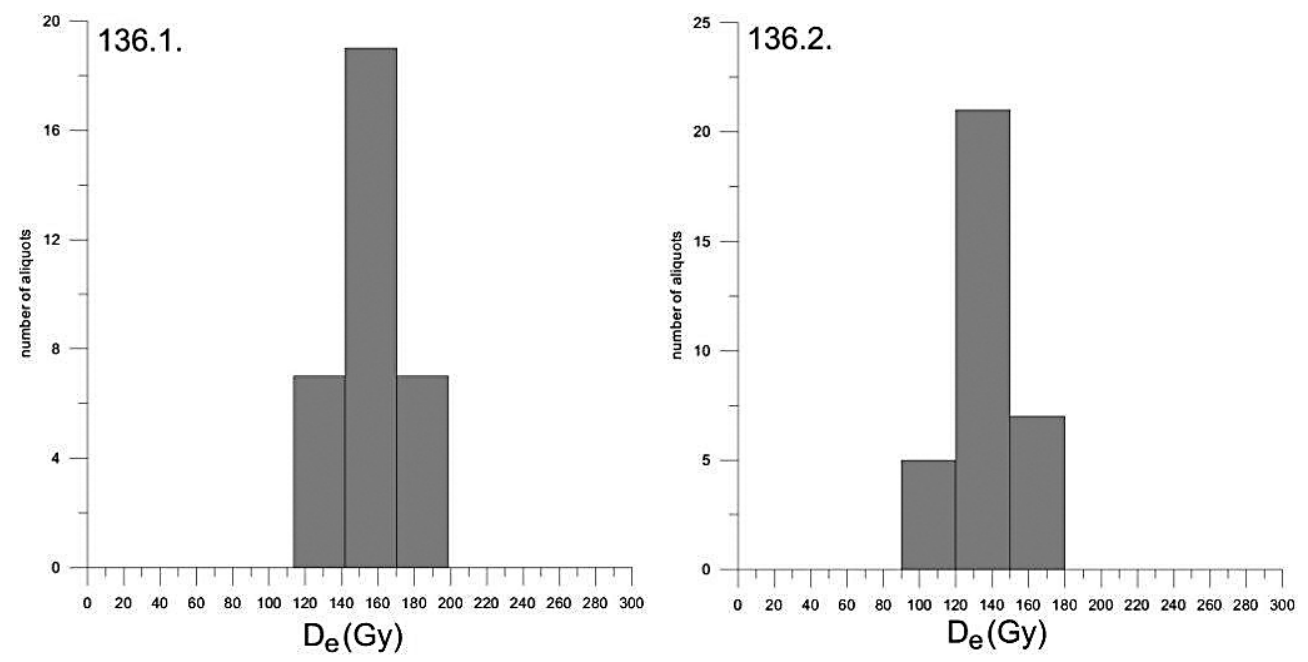

Figure 9 - Langeais La Roche-Cotard II. Histogrammes OSL pour les échantillons 136.1 et 136;2.

Figure 9 - Langeais. La Roche-Cotard II. OSL histograms 136.1 and 136;2.

\begin{tabular}{|c|c|c|c|c|c|c|c|c|c|c|c|c|c|c|c|c|}
\hline \multirow{2}{*}{$\begin{array}{c}\text { sample } \\
136.1 .\end{array}$} & \multirow{2}{*}{$\begin{array}{c}\begin{array}{c}\text { depth } \\
\text { (cm) }\end{array} \\
180\end{array}$} & \multicolumn{2}{|c|}{$\begin{array}{c}\mathrm{U} \\
(\mathrm{ppm})\end{array}$} & \multicolumn{2}{|c|}{$\begin{array}{c}\text { Th } \\
(\mathrm{ppm})\end{array}$} & \multicolumn{2}{|c|}{$\begin{array}{c}\mathrm{K} \\
(\%)\end{array}$} & \multicolumn{3}{|c|}{$\begin{array}{c}\text { equivalent } \\
\text { dose }(G y)\end{array}$} & \multirow{2}{*}{\begin{tabular}{|l|}
$\mathbf{n}$ \\
33 \\
\end{tabular}} & \multirow{2}{*}{\begin{tabular}{|c|}
$\begin{array}{c}\text { w.c. } \\
\text { \% }\end{array}$ \\
10,4 \\
\end{tabular}} & \multicolumn{2}{|c|}{$\begin{array}{c}\text { dose rate } \\
(\mathrm{Gy} / \mathbf{k a})\end{array}$} & \multicolumn{2}{|c|}{$\begin{array}{c}\text { OSL age } \\
\text { (ka) }\end{array}$} \\
\hline & & 1,29 & $\pm 0,02$ & 45 & 0,10 & 1,31 &, 01 & 56,15 & & 2,89 & & & 2,06 & 0,15 & 75,6 & 5,8 \\
\hline 136.2. & 160 & 1,22 & $\pm 0,02$ & 5,49 & $\pm 0,09$ & 1,43 & $\pm 0,01$ & $138,18=$ & \pm 2 & 2,50 & 33 & 13,3 & 2,10 & $\pm 0,15$ & 65,7 & \pm 5 \\
\hline
\end{tabular}

n: number of aliquots, w.c.: water content

Tableau 2 - Langeais La Roche-Cotard II. Ages OSL calculés dans le cas d'une teneur en eau moyenne des échantillons.

Tableau 2 - Langeais. La Roche-Cotard II. OSL ages calculated with the average equivalent dose of the samples. 


\section{Acknowledgements}

For helpful discussion Prof. Andrew Murray (Aarhus University, Denmark) is gratefully acknowledged. In the Geological and Geophysical Institute of Hungary Attila Nagy, Mariann Török-Sinka and Péter Cserny are thanked for gamma spectrometry measurements, Judit Füri and Miklósné Bátori for the help in luminescence measurements and sample preparation, and Zsolt Horváth for the measurement of water content.

\section{Bibliography}

ADAMIEC G., AITKEN M.J. 1998 - Dose-rate conversion factors: update. Ancient TL 16, p. 37-50.
AITKEN M.J. 1998 - An Introduction to Optical Dating. The Dating of Quaternary Sediments by the Use of Photonstimulated Luminescence. Oxford University Press, Oxford.

THAMÓ-BOZSÓ E., NAGY A., FÜRI J., TÖRÖKSINKA M., BÁTORI M-NÉ, HORVÁTH ZS., CSERNY P. 2015 - Results of luminescence dating of samples from La Roche-Cotard (France). Manucript. Geological and Geophysical Institute of Hungary, Department of Geochemistry and Laboratories. Inedit report

WINTLE A., MURRAY A.S. 2006 - A review of quartz optically stimulated luminescence characteristics and their relevance in single-aliquot regeneration dating protocols. Radiation Measurements 41, p. 369-391. 
